\title{
Long-running Transactions: semantics, schemas, implementation
}

\author{
Sotiris Moschoyiannis, Member, IEEe, Amir R Razavi, Yongyan Zheng, Paul Krause \\ Department of Computing, Faculty of Engineering and Physical Sciences, \\ University of Surrey, Guildford, Surrey, GU2 7XH \\ e-mail : \{s.moschoyiannis | a.razavi | y.zheng | p.krause\}@surrey.ac.uk
}

\begin{abstract}
In this paper we describe a formal model for the distributed coordination of long-running transactions in a Digital Ecosystem for business, involving Small and Medium Enterprises (SMEs). The proposed non-interleaving model of interaction-based service composition allows for communication between internal activities of transactions. The formal semantics of the various modes of service composition are represented by standard $x m l$ schemas. The current implementation framework uses suitable asynchronous message passing techniques and reflects the design decisions of the proposed model for distributed transactions in digital ecosystems.
\end{abstract}

Index Terms-service orchestration, concurrency, vector semantics, schema, message passing.

\section{INTRODUCTION}

The concept of a Digital Ecosystem for business emerges as a key feature in the era of the Digital Economy. The importance of a pool of rare species in sustaining (innovation in) an ecosystem is well understood in the studies of biodiversity. In a digital ecosystem for business, SMEs can be seen to play the role of 'rare species'.

A business transaction between SMEs in a Digital Ecosystem can be either a simple usage of a web service or a mixture of different levels of composition of several services from various service providers. The specification of a business transaction may allow it to be completed over a period of minutes, hours, or even days - hence, the term long-lived or long-running transaction. The execution of a long-running transaction corresponds to conducting a business activity and typically comprises a number of subtransactions or activities that involve the execution of a number of underlying services.

This makes Service-Oriented Computing (SOC) [1], whose goal is to enable applications from different providers to be offered as services that can be used, composed and coordinated in a loosely-coupled manner, the prevalent computing paradigm in a digital business ecosystem. The actual architectural approach of SOC is called SOA [2] and is particularly applicable when multiple distributed applications are running on varied technologies and platforms need to communicate with each other.

Business transactions in a B2B context typically involve interactions between multiple partners, either service providers, or service consumers, or both. This requires that all partners behave in a coordinated manner - partners must follow some protocol to execute a transaction effectively.

Conventional transaction models such as Sagas [3] or the more recent models targeting web services Web Services
Transactions (WS-Tx) [4] and Business Transaction Protocol (BTP) [5] seem to be geared towards centralised control (using the WS-Coordination framework [6]) which means some knowledge of the internal build-up of the participants is required. This violates the primary requirement of SOA for loosely-coupled services and may not be acceptable or even possible in a business environment - in a digital ecosystem involving communities of SMEs this raises a barrier for the adoption of SOA as it violates local autonomy. Part of the problem seems to be that these frameworks lack a formal model for the coordination of the underlying services involved in the execution of a long-running transaction.

In this paper we give a formal description of longrunning multi-service transactions in terms of the distributed orchestration of the underlying service executions. The formal semantics, in the first instance, is aimed at describing the behavioural patterns services should follow in order to increase confidence in a successful outcome. The coordination of a transaction in our approach amounts to interaction-based composition of the underlying services and thus we advocate the use of XML schemas that reflect the message-based protocol used in a transaction. Finally, we describe a prototype implementation of our ideas on the distributed coordination of long-running transactions and the associated structural schemas.

The remainder of this paper is structured as follows. Section II outlines earlier work on a distributed transaction model for digital ecosystems. Section III introduces the formal description of a transaction in terms of its underlying service executions. Section IV provides a XML schema that captures the formal model for service coordination and reflects the resulting transaction structure. The prototype implementation of the transaction structure schema is then presented in Section V. The paper finishes with some concluding remarks and ideas for future work given in Section VI.

\section{Distributed Transaction Model FOR DES}

In earlier work [7], we have described a distributed transaction model for networked organisations in the digital ecosystem paradigm. Here we outline the basic ideas.

It is often the case that internal activities of a transaction need to share results before the termination of the transaction (transaction commit). More generally, dependencies may exist between activities inside a transaction due to the required ordering on service invocations (e.g. book a hotel 
only after booking the flight) or due to the sharing of data (one uses the results of another). The use of log structures, given in the form of directed graphs, has been described in [7], [8] for capturing the internal and external dependencies of a transaction. The benefit of using these lightweight logs is that each platform coordinator only needs to have knowledge about its services and their dependencies to other coordinators' services - it needs to know only what happens before and after its own services. Due to space limitations we omit further details.

In our model a transaction is represented by a tree structure that allows us for nested subtransactions and exemplifies the local coordination that is required for the services involved to be performed in unison.

Drawing upon the latest work on an extended serviceoriented architecture for a business environment [2], we have considered five different types of coordinators which allow for various modes of service interaction in our model. In the transaction tree of Fig. 1, the services s3 and s4 for example are children of a sequential coordinator and hence $\mathrm{s} 4$ can only be executed after $\mathrm{s} 3$. In other words the execution of $s 4$ is dependent on the (successful) execution of $s 3$, e.g. $\mathrm{s} 4$ uses use the results released by s3.

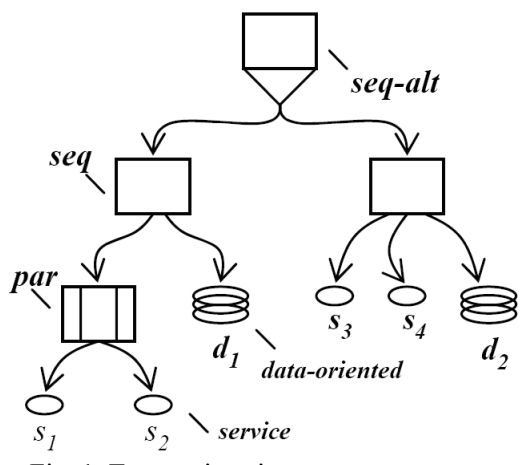

Fig. 1 Transactions in a tree structure

The scenario described in Fig. 1 has appeared in [7] and has been simplified here, nevertheless the transaction in question is complicated enough to illustrate the key ideas of our formal modelling approach.

\section{Transaction Context Schema}

The DE nested transaction model can be represented in a context schema. This is used by the Initiator of a transaction to specify the different levels of compositions of the underlying services. The schema also shows the boundaries and requirements of the model and the need for a formal language to describe transactions, which will be given in Section IV. Fig. 2 shows such a schema (the Netbeans 5.5.1 source can be found following [9]).

The nested transaction structure is imposed in the schema using the 'Coordinator' element which can include a service composition ('Composition' element in the schema) or usage of a simple web service ('WebService') including a purely data-oriented web service (a leaf of the tree). Each service composition ('Composition') has a type ('CompositionType'), which determine the mode of the interaction-based composition (sequential, parallel or alterna- tive). It has two or more 'Coordinator' elements which introduce recursion - another service composition or a simple web service. This recursive definition can generate an XML representation of the transaction tree. In the following sections we interpret this context schema into a formal language which allows for an XML presentation of the semantics of a transaction can be implemented in different platforms.

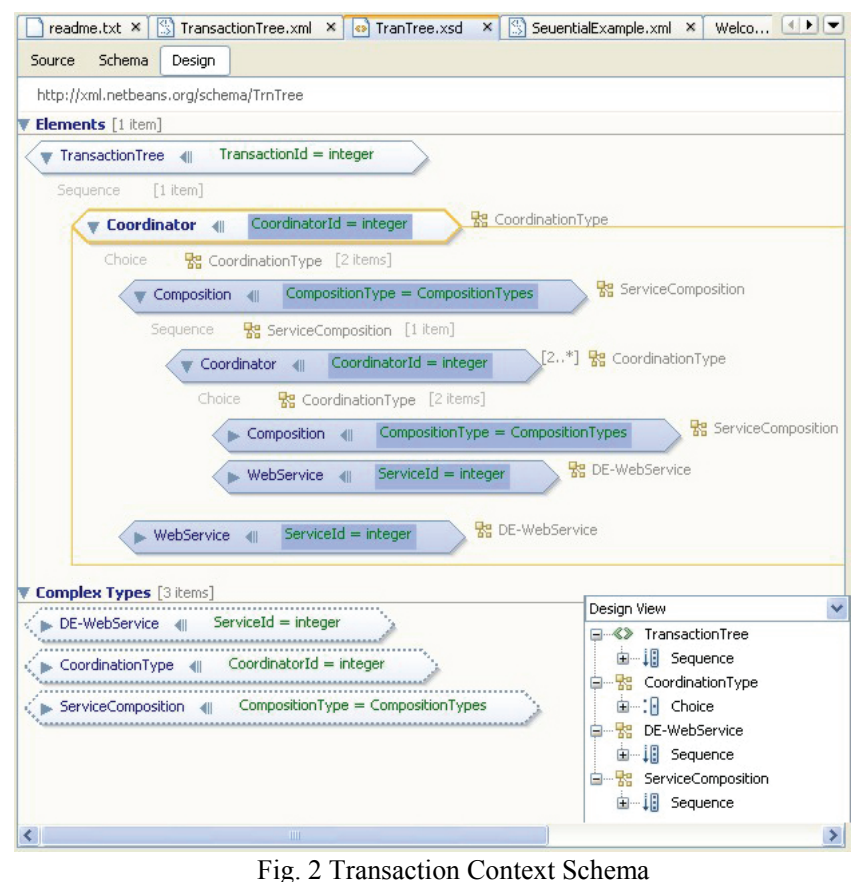

\section{A Formal LANGUAGe FOR Transactions}

In this section we introduce a formal language for describing long-running transactions. Apart from the dependencies between service executions, there is a high degree of concurrency in a transactional environment since real problems require a number of activities to take place in parallel. For this reason we will find the general theory of noninterleaving representation of parallel behaviour found in [10] of great use in what follows. As mentioned before, our objective is to get a thorough understanding of the behaviour the underlying service compositions need to exhibit for a successful outcome of the transaction as a whole.

The semantics is intended to describe the behaviour of a transaction in terms of its services at the deployment level, but not the low-level computations performed by the services themselves. Note that services are offered in a digital ecosystem for business from different service providers and it is important that we defer from interfering with the local state of the service execution. This means it is appropriate to consider that any action within the transaction model has no significant duration, in the sense that (i) it either occurs as a whole or not at all; (ii) it occurs either wholly before, or wholly after, or wholly in parallel with, every other action.

A transaction $T$ is associated with a set of leaves or $a c$ cess points $L$ which consists of a set of basic services $S$, a set of data-oriented coordinators $D$ and a set of delegation coordinators $D l g$. Hence, $L=S \cup D \cup D l g$. We further require that the sets $S, D$ and $D l g$ are pairwise disjoint. 
Actions within a long-running transaction take place on the leaves and therefore each leaf is in turn associated with a set of actions that may occur on that leaf, depending on its nature. We denote this set by $\mu(l), l \in L$, and require that

$$
\bigcup_{l \in L} \mu(l) \subseteq M \text {. }
$$

As can be seen in Fig. 1 a transaction has a number of activation points, namely the leaves of the corresponding transaction tree. Thus, instead of modelling a transaction by a sequential process that would generate a trace of a single access point, we model the behaviour of a transaction by considering a set of such sequences at the same time, one sequence for each leaf. This draws upon Shields'vector languages [9] and allows us to capture what is happening on each access point of a long-running transaction.

Transaction vectors. Let $T$ be a transaction. We define $V_{T}$ to be the set of all functions $\underline{v}: L \rightarrow M^{*}$ such that $\mathrm{v}(1) \in \mu(1)^{*}$.

By $\mu(l)^{*}$ we denote the set of finite sequences over $\mu(l)$. Mathematically, the set $V_{T}$ is the Cartesian product of the sets $\mu(l)^{*}$, for each $l$. Effectively, transaction vectors are $n$-tuples of sequences where each coordinate corresponds to a leaf in the transaction tree (hence, $n$ is the number of leaves) and contains a finite sequence of actions that have occurred on that leaf or access point.

When an action occurs on a leaf of the transaction tree, that is to say when an action associated with some subtransaction takes place on an access point, it appears on a new transaction vector and at the appropriate coordinate. For example, the vector $\left(s_{1}, \Lambda, \Lambda\right)$ describes that portion of behaviour of the transaction in which an action $s_{1}$ (e.g. service invocation) has taken place on the corresponding service allocated to the first coordinate. The vector $\left(s_{1}, s_{2}, \Lambda\right)$ describes that portion of behaviour in which both $s_{1}$ and $s_{2}$ have happened on the corresponding services while the vector $\left(s_{1} s_{3}, s_{2}, \Lambda\right)$ describes an occurrence of $s_{1}$ and an occurrence of $s_{3}$ on the service corresponding to the first coordinate, and an occurrence of $s_{2}$ on the second coordinate. Nothing has happened on the service corresponding to the third coordinate.

In this sense, each transaction vector provides a snapshot of behaviour in which the transaction has executed the actions appearing on the vector's coordinates - it describes what actions have already occurred and on which part of the transaction tree. Fig. 3 shows the xml schema types for building a single transaction vector. Each transaction vector comprises a specific number of 'access points' (five in our example), and each access point corresponds to a service, a data-oriented coordinators or a delegation coordinator, all presented as 'DE-WebServices' in our xml schema.

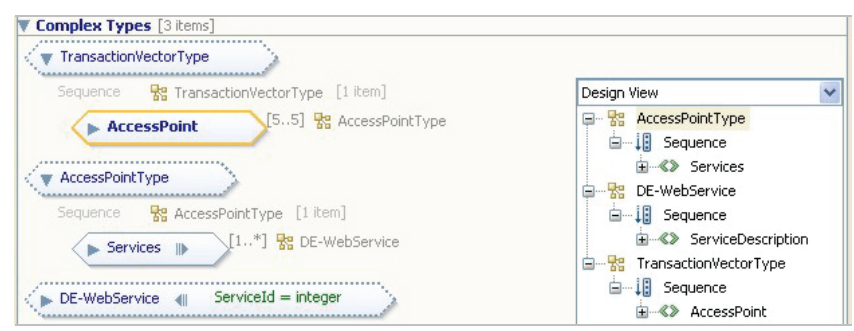

Fig. 3 A single vector type (xml schema presentation)
It can be seen from the example given above that there is already an ordering among actions on a particular access point or subtransaction, e.g. $s_{1}$ followed by $s_{3}$. This vectorbased behavioural description of transactions can also capture the orderings between different subtransactions, which amounts to actions appearing on different vector coordinates. This requires however a more careful consideration of the mathematical properties of such vectors which we briefly describe in the sequel.

Before examining the mathematical properties of our construction so far, we introduce a specific kind of transaction vector, which is used in our model to describe actions (events or activations) within a transaction.

Column vectors. Let $T$ be a transaction and $V_{T}$ its set of transaction vectors. We define

$$
A_{T}=\left\{\underline{a} \in V_{T} \backslash\left\{\underline{\Lambda}_{T}\right\}: l \in L \Rightarrow|\underline{a}(l)| \leq 1\right\}
$$

where $|x|$ denotes the length of sequence $x$. We refer to elements of $A_{T}$ as column vectors.

Thus, column vectors are themselves transaction vectors, but have the additional constraint that each of their coordinates is either the empty sequence or a single action. For example, the vector $\left(s_{1}, \Lambda, \Lambda\right)$ represents the occurrence of an action $s_{l}$ on the service associated with the first coordinate.

We will use the term transaction language to refer to a subset $V$ of all possible vectors $V_{T}$ formed over a given transaction $T$. Hence, a transaction $T$ comes with a language $V$, where $\mathrm{V} \subseteq \mathrm{V}_{\mathrm{T}}$. The idea is that the particular set of transaction vectors for a specific transaction expresses the ordering constraints necessary in the corresponding service orchestration.

We have seen that transaction vectors are essentially tuples of sequences. This can be exploited in defining operations on vectors in terms of well-known operations on sequences.

Operations on vectors. For $\underline{u}, \underline{v} \in V_{T}$, we define

- $\underline{u} \cdot \underline{v}$ to be the unique vector $\underline{w}$ such that $\underline{w}(l)=\underline{u}(l) \cdot \underline{v}(l)$, for each $l \in \mathrm{L}$ (concatenation)

- $\underline{u} \leq \underline{v}$ iff $\underline{u}(l) \leq \underline{v}(l)$, for each $l \in \mathrm{L}$ (prefix ordering)

- $g l b(\underline{u}, \underline{v})$ to be the vector $\underline{w}$ such that $\underline{w}(l)=\min (\underline{u}(l), \underline{v}(l))$, for each $l \in \mathrm{L}$

- lub( $\underline{u}, \underline{v})$ (if it exists) to be the vector $w$ such that $\underline{w}(l)=\max (\underline{u}(l), \underline{v}(l))$, for each $l \in \mathrm{L}$

- if $\underline{u} \leq \underline{v}$, then we define $\underline{v} / \underline{u}$ to be the unique element $\underline{z} \in \mathrm{V}_{\mathrm{T}}$ such that $\underline{u} . \underline{z}=\underline{v}$ (right-cancellation)

Thus, the operation of concatenation on vectors is defined in terms of the concatenation of sequences appearing on their respective coordinates. For example,

$$
\left(s_{1} s_{3}, s_{2}, \Lambda\right) .\left(\Lambda, s_{4}, \Lambda\right)=\left(s_{1} s_{3}, s_{2} s_{4}, \Lambda\right)
$$

The ordering amongst vectors is defined in terms of the usual prefix ordering operation on sequences appearing on their coordinates. For example, $\left(s_{1}, s_{2}, \Lambda\right) \leq\left(s_{1} s_{3}, s_{2}, \Lambda\right)$ since $s_{1} \leq s_{1} s_{3}$ and $s_{2} \leq s_{2}$ and $\Lambda \leq \Lambda$. In other words, the second vector 'wins' on the first coordinate (since it has a sequence of greater length in this coordinate) while the two vectors draw on all other coordinates.

It is not hard to see that some vectors will be incomparable. It turns out that such vectors describe either parallel 
or alternative behaviours of the transaction in question, and this will be further discussed in the following sections.

The operations $g l b()$ and $l u b()$ give the greatest lower bound and the least upper bound, respectively of $\underline{u}, \underline{v} \in V_{T}$, in the usual sense of lattices and domain theory [11]. As we will see, these operations are central to the treatment of concurrency in our approach.

The right cancellation operator '/" says that if $\underline{u}$ is a transaction vector describing an initial part of the behaviour described by $\underline{v}$ so that $\underline{u} \leq \underline{v}$, then $\underline{v} / \underline{u}$ is the 'continuation' of $\underline{u}$ that extends it to $\underline{v}$. This operation is central to the treatment of compensations in our approach. We return to this discussion in the concluding section of the paper.

It is important to stress the fact that all operations on vectors are performed coordinate-wise and this simplifies the proofs but also makes the formal model feasible for implementation.

Transaction vectors can be seen to be built up from the empty vector $\underline{\Lambda}_{T}$ by a series of concatenations with column vectors that represent actions. In fact, in describing the behaviour of a transaction we are interested only in those vectors describing (orderings of) actions that we expect the transaction to engage in during the course of its execution. This is the subset of all possible transaction vectors, over a given $T$, we referred to as transaction language.

\section{A. Sequential service compositions}

The prefix ordering relation on transaction vectors can be viewed as an ordering on partial executions, where each vector corresponds to that portion of behaviour in which the transaction has already engaged in the actions appearing on its coordinates. This can be expressed more succinctly by saying that $\underline{u} \leq \underline{v}$ in a transaction language means that $\underline{u}$ is an earlier part of behaviour leading to $\underline{v}$.

A more careful examination of the mathematical construction shows that we can say more than that. Indeed, we find it useful to determine immediate predecessors (or successors) of a transaction vector.

Cover. Suppose that $\underline{u}, \underline{v} \in V \subseteq V_{T}$. We say that $\underline{v}$ covers $\underline{u}$ in $V$, and we write $\underline{u} \triangleleft_{V} \underline{v}$, if (i) $\underline{u} \leq \underline{v}$ and $\underline{u} \neq \underline{v}$ and (ii) If $\underline{z} \in V$ such that $\underline{u} \leq \underline{z} \leq \underline{v}$, then $\underline{z}=\underline{u} \vee \underline{z}=\underline{v}$.

Thus, whenever $\underline{u} \leq \underline{v}$, and we also have that $\underline{u} \triangleleft \underline{v}$, then the last actions that went into forming each vector have occurred in sequence - one after the other. This allows to model sequential dependency among services inside a transaction. Recall the example of Fig. 1 where service $s_{3}$ feeds service $s_{4}$.

Fig. 4 presents the corresponding schema for sequential service composition. The 'TransactionVectors-Type' has a TransactionVector element which includes a single transaction vector (e.g. describing the service invocation $s_{3}$ ) and the immediate successor (describing the service invocation $s_{4}$ ). The latter is captured by the 'FollowedBy' element. The type of 'SequentialComposition' simply includes a single transaction vector followed by another transaction vector.

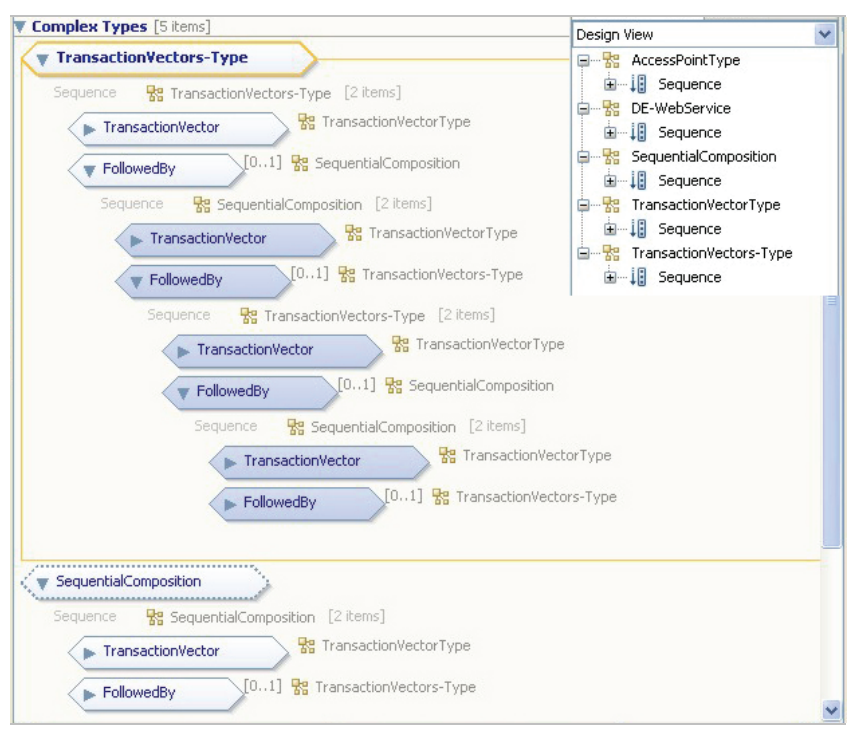

Fig. 4 Sequential service composition (xml schema presentation)

\section{B. Parallel service compositions}

Our approach towards modelling concurrent actions, actions that can happen in parallel, draws upon the concepts in Shields' vector languages [10] and Mazurkiewicz trace languages [12] where concurrent events are considered as being unordered, in contrast to CSP trace theory where it is assumed that observations are sequential in nature and concurrent events are understood to occur in either order (nondeterministic interleaving).

The treatment of concurrency within our formal model of transactions thus takes up on non-interleaving models of concurrency, which introduce additional structure into formal languages in order to describe non-sequential behaviour. The additional structure is given in terms of an independence relation over action symbols, which describes potential concurrency.

Drawing upon the extension of the independence relation 1 to behaviour vectors in [9], the notion of independence between actions in Mazurkiewicz traces can be readily interpreted into transaction vectors in our approach.

Independence. For $\underline{u}, \underline{v} \in V \subseteq V_{T}$, we define

$$
\underline{u} \text { ind } \underline{v} \Leftrightarrow \forall l \in L: \underline{u}(l)>\Lambda \Rightarrow \underline{v}(l)=\Lambda
$$

This definition says that two transaction vectors are independent if the behaviours they describe concern distinct services (correspond to activation on different leaves of the corresponding transaction tree). This means that the behaviours described by $\underline{u}$ and $\underline{v}$ may occur independently.

In the case of column vectors, independence captures the fact that actions appearing in one vector may occur independently of those appearing in the other. If in addition the vectors representing these actions are adjacent in an expression (of the series of concatenations that went into forming the corresponding transaction vectors), then the actions are concurrent. Thus, whenever two actions are independent and are both enabled (can both occur at some point, after some behaviour) then, their corresponding column vectors commute, i.e. $\underline{a}_{1} \cdot \underline{a}_{2}=\underline{a}_{2} \cdot \underline{a}_{1}$, and in the resulting behaviour the two actions are concurrent.

The parallel service composition schema is depicted in Fig. 5. A 'ParallelComposition' consists of two or more (up 
to the number of its access points, in this example 5) transaction vectors, followed by a 'TransactionVectors-Type', which imposes that all transaction vectors describing parallel executions have one immediate successor (their $l u b($ ) identified in the schema by the 'FollowedBy' element) and the concurrent service invocations can happen on different access points (recall the independence relation - two actions are independent, and potentially concurrent, if they engage different access points).

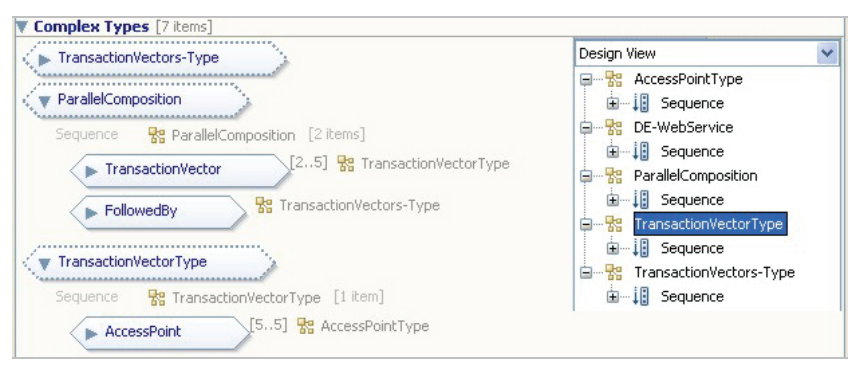

Fig. 5 Parallel service composition (xml schema presentation)

\section{Alternative service compositions}

Based on the prefix ordering between transaction vectors in the set $V$ we may also model a choice between actions. That is, actions which are mutually exclusive in that occurrence of one excludes occurrence of the other.

In discussing concurrent actions in a long-running transaction, we saw that the two incomparable transaction vectors represent concurrent behaviour. The vector they both cover is in fact their greatest lower bound and is obtained by applying the operation $g l b()$ given earlier. The fact the two incomparable vectors represent concurrent actions is only because they are bounded above in the set (by the transaction vector which is their $\operatorname{lu} b()$ and is sitting on top of the lozenge). Whenever this latter requirement does not hold we may talk about events in conflict.

It might be instructive to make the distinction in terms of pictures and associated Hasse diagrams. In the diagram of Fig. 1, $s_{1}$ and $s_{2}$ are sequential ( $s_{2}$ can only be invoked after s1) in Fig.6(i) while there is a choice between them (alternative) in Fig.6(ii) and they are concurrent in Fig.6(iii).

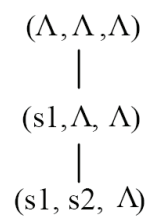

(i)

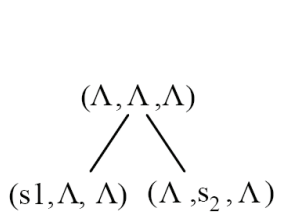

(ii)

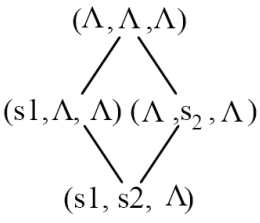

(iii)
Fig. 6 Order structure of transaction vectors

Notice that the set of vectors in (i) does not include $(\Lambda$, $s_{2}, \Lambda$ ), which means that $s_{2}$ never occurs before $s_{1}$; in (ii) it does not include $\left(s_{1}, s_{2}, \Lambda\right)$ which means there is no valid behaviour of the transaction processing system in which both $s_{1}$ and $s_{2}$ have taken place; in (iii) it includes all four vectors, which means that $s_{1}, s_{2}$ and both $s_{1}$ and $s_{2}$ are all valid observations of the behaviour of the transaction system in which $s_{1}$ and $s_{2}$ happened concurrently. This is indicated by the familiar lozenge shape that exhibits the charac- teristic structure of a finite lattice.

In further explanation, the vector sitting at the bottom of the lozenge is the $g l b()$ of the two incomparable vectors $\left(s_{1}, \Lambda, \Lambda\right)$ and $\left(\Lambda, s_{2}, \Lambda\right)$ sitting at the middle of the lozenge represent while the vector at the top is their $\operatorname{lub}()$. The lozenge as a whole describes that part of behaviour of the transaction in which $s_{1}$ and $s_{2}$ happened concurrently, as indicated by the vector at its top. Further details on how the ordering relations between actions are manifested in the resulting order structure of the resulting set of vectors can be found in [13].

Fig. 7 presents the alternative service composition schema. An 'AlternativeComposition' consists of two or more (up to the number of its access points, here 5) alternative transaction vectors. Notice that in this case there is no 'FollowedBy' element as vectors describing alterantive service deployment do not lead to a common vector, i.e. do not have a $\operatorname{lu} b()$ in the formal language.

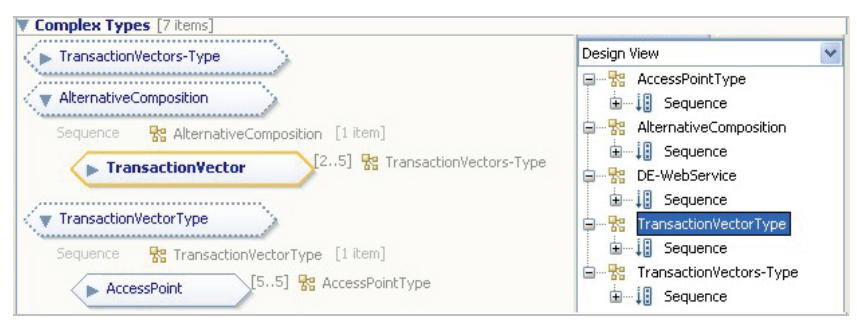

Fig. 7. Alternative service composition (xml schema presentation)

\section{IMPLEMENTATION}

We have used JAX-WS 2.1, Java 5, and Netbeans IDE 5.5.1 in our implementation framework. The JAX-WS is a new standard for message passing, it supports both synchronous and asynchronous message passing by the polling model and the call-back model, respectively. In the polling model, the client continuously polls the service response. In the call-back model, the client creates a call-back handler. JAX-WS is shown to perform better than JAX-RPC in certain aspects [14]. Furthermore, JAX-WS supports static and dynamic generation of web service client stubs.

In our implementation, the JAX-WS call-back message passing and dynamic client stub generation are used. The call-back message passing is suitable for asynchronous message passing, which is important for long term transactions. The dynamic WS client creation enables web service invocation chains. Three participants are required in order to make a service $\mathrm{X}$ work: 1) $\mathrm{X}$ web service; 2) $\mathrm{X}$ TransactionAgent web service; 3 ) the client stubs for the $X$ web service. The dependencies between web services are captured in a XML file, as discussed before.

From the transaction vector schema, we can create different transaction scenarios (as xml file). The generated xml file shows the various states of a transaction for a specific scenario. We have designed a software agent coordinator which can perform the transaction vector in fully distributed manner. As a case study we analyse a simple transaction, with a sequential service composition, which involves the interactions shown in the Sequence diagram of Fig. 8. 


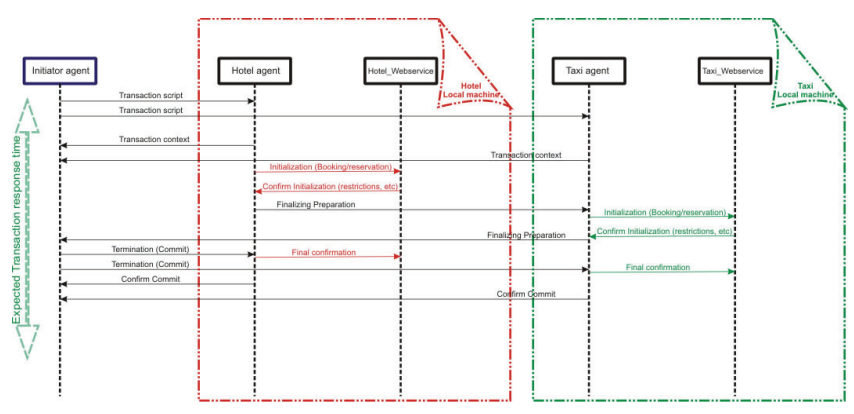

Fig. 8 A simple transaction with a sequential service composition

Our simple transaction scenario involves two participants, located at two different SMEs (Hotel and Taxi services). The initiator agent has an AgentHelper that communicates with the TransactionAgents. The transaction involves four players: HotelTransactionAgent (HTA; local coordinator of the Hotel), HotelService (webservice of the hotel), TaxiTransactionAgent (TTA; local coordinator of taxi), and TaxiService (webservice of the taxi). The AgentHelper plays the Initiator role and it starrts the transaction by sending messages (setting the transaction context) to both HTA and TTA. This specifies the first web service should be deployed on the first access point (HTA) and the second will be deployed only after receiving the successful confirmation of the first (sequential service composition).

The following information about the required distributed coordination can be derived: 1) the AgentHelper will know that after it initiates the HTA and TTA, it needs to wait for a response from the TTA for the final preparation status; 2) the HTA will know that it depends on TTA so it needs to send a message to TTA asking for final preparation status; 3 ) the TTA will know that it gets a request message from the HTA and then sends its response message back to the AgentHelper for the final preparation status. Fig 9 shows the performance analysis of the case study in terms of CPU performance, memory usage, and thread usage when the client performs a commit transaction. The Netbeans 5.5.1 source is available following [9].

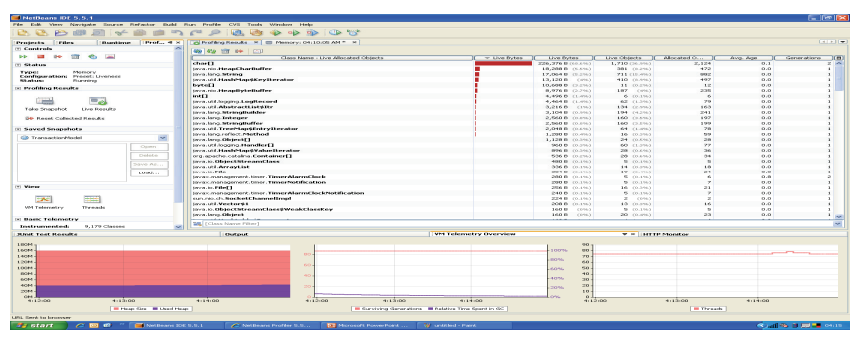

Fig. 9 Run time behaviour of the scenario

\section{CONCLUSIONS AND FUTURE WORK}

We have described an integrated view of various aspects of a transaction model for digital ecosystems for business. To harness the complexity in this highly dynamic environment and increase confidence in a successful outcome, a thorough understanding of the underlying service execu- tions and their dependencies is essential. This has been given by a formal model that can be represented by standard $\mathrm{xml}$ schemas and be used to guide the implementation.

We laid the foundations for the formal modelling of long-running transactions in terms of the underlying service executions which are orchestrated locally. The trueconcurrent formal semantics allows internal activities of a transaction to communicate and can capture parallelism, unlike other approaches [15]. An extension to address partial results (released outside a transaction), based on [16], and compensating actions is currently under investigation at both the modelling and implementation levels. Preliminary results [17] are encouraging.

The current implementation framework is independent of the underlying network supporting the transactions. Work is in progress on integrating the implementation for the transaction model with the P2P network [17].

\section{Acknowledgements:}

This work was supported by the EU-FP6 funded project OPAALS Contract No 034824

\section{REFERENCES}

[1] M. P. Papazoglou. Service-Oriented Computing: Concepts, Characteristics and Directions. In Proc. WISE'03, IEEE, pp. 3-12, 2003.

[2] M. P. Papazoglou, P. Traverso, S. Dustdar et al. Service-Oriented Computing Roadmap. In Dagstuhl Seminar Proc. 05462, ServiceOrienrted Computing (SOC), pp. 1-29, 2006.

[3] H. Garcia-Molina and K. Salem. Sagas. In ACM SIGMOD, pp. 249259, 1987.

[4] L.F. Cabrera, G. Copeland, J. Johnson and D. Langworthy. Coordinating Web Services Activities with WS-Coordination, WSAtomicTransaction, and WS-BusinessActivity. January 2004. Available: http://msdn.microsoft.com/webservices/default.aspx

[5] L.F. Cabrera, G. Copeland, W. Cox et al. Web Services Business Activity Framework (WS-BusinessActivity). August 2005. Available http://www128.ibm.com/developerworks/webservices

[6] L.F. Cabrera, G. Copeland, M. Feingold et al. Web Services Coordination (WS-Coordination). August 2005. Available http://www-128 .ibm.com/developerworks/webservices/library/specification/ws-tx

[7] A. Razavi, S. Moschoyiannis, P. Krause. A Coordination Model for Distributed Transactions in Digital Ecosystems. In IEEE Digital Ecosystems and Technologies (IEEE-DEST'07), 2007.

[8] A. Razavi, P. J .Krause and S. K. Moschoyiannis. Digital Business Ecosystem (DBE) Project Deliverable D24.5: DBE Distributed Transaction Model, 2006.

[9] http://www.computing.surrey.ac.uk/personal/st/S.Moschoyiannis

[10] M. W. Shields. Semantics of Parallelism. Springer-Verlag, 1997.

[11] B.A. Davey and H.A. Priestley. Introduction to Lattices and Order, Cambridge University Press, 1990.

[12] A. Mazurkiewicz. Basic Notions of Trace Theory. In Linear time, Branching Time and Partial Orders in Logics and Models of Concurrency, LNCS, 354, pp. 285-363, Springer, 1988.

[13] S. Moschoyiannis. Specification and Analysis of Component-Based Software in a True-Concurrent Setting. PhD Thesis, UniS, 2005.

[14] http://java.sun.com/developer/technicalArticles/WebServices/high_pe rformance/

[15] M. Butler, A.C.R. Hoare, C. Ferreira. Trace Semantics for LongRunning Transactions. In 25 Years of CSP, LNCS 3525, pp. 133-150, 2005

[16] S.Moschoyianis, M.W. Shields. A Set-Theoretic Framework for Composition. Fundamenta Informaticae 59(4):373-396, 2004.

[17] A. Razavi, S. Moschoyiannis and P. J. Krause "Preliminary Architecture for Autopoietic P2P Network focusing on Hierarchical SuperPeers, Birth and Growth Models." OPAALS project Deliverable D3.2, 2007 - available at: http://files.opaals.org/OPAALS 